\title{
Gay, bisexual, and other men who have sex with men: time to end the fixation with HIV
}

In the sixth paragraph (starting Chemsex) of this Editorial (BMJ 2016;354:i4739, doi:10.1136/bmj.i4739), the second sentence should say, "It is associated with anal sex without a condom."
We would like to apologise for any confusion that this omission may have caused. 